\title{
Relação da prática de exercícios físicos e fatores associados às regulações motivacionais de adolescentes brasileiros
}

\author{
Relationship between physical exercise practice and associated factors to \\ motivational regulations of Brazilians adolescents
}

\author{
R.B. Silva, T.S. Matias, M.S. Viana, A. Andrade
}

ARTIGO ORIGINAL | ORIGINAL ARTICLE Com base na Teoria da Autodeterminação (TAD; Deci \& Ryan, 1985), o presente estudo objetivou investigar as relações entre a prática de exercícios físicos e suas regulações motivacionais, bem como os fatores associados a estas em estudantes adolescentes. Realizou-se um estudo transversal com a participação de 471 adolescentes de 14 a 18 anos de idade, estudantes de uma escola pública estadual no município de Florianópolis/SC, Brasil. Questionários foram utilizados para a realização da pesquisa e os dados foram tratados com estatística descritiva e inferencial. Meninos foram mais ativos e mais autodeterminados para a prática de exercícios físicos do que as meninas. Para ambos os sexos as regulações motivacionais mais internas, bem como o índice de autodeterminação, estiveram associados positivamente com a prática de exercícios físicos. Meninas com maior idade e com presença de sobrepeso apresentaram maior regulação identificada. Entre os meninos, os estudantes com sobrepeso mostraram-se mais amotivados e menos motivados intrinsecamente. De maneira geral, adolescentes que estudam no período vespertino apresentaram um maior índice de autodeterminação. Conclui-se que adolescentes mais autodeterminados praticam mais exercícios físicos regularmente. Destacam-se ainda os fatores associados à motivação e a existência de diferenças entre meninas e meninos nas relações entre as variáveis investigadas.

Palavras-chave: adolescente, atividade física, motivação, teoria da autodeterminação

ABSTRACT

Based on Self-Determination Theory (SDT; Deci \& Ryan, 1985), this study investigated the relationship between physical exercise, motivational regulations and associated factors in adolescents. We conducted a cross-sectional study involving 471 adolescents aged 14 to 18 years old, students at a public school in Florianópolis/SC, Brazil. Questionnaires were used to conduct the survey and the data was analyzed by descriptive and inferential statistics. Boys were more active and more self-determined for physical exercise than girls. For both genders the internal motivations and the self-determination were positively associated with physical exercise. The older and overweight girls had higher identified regulation. Among boys, students who are overweight were more amotivated and less intrinsically motivated. All students, adolescents who are studying in the afternoon period showed a higher baseline of self-determination. The study concluded that adolescents more self-determined did more physical exercise regularly. Also the study showed that there are factors associated with the motivational regulations and that there are differences between girls and boys.

Keywords: adolescents, physical activity, motivation, self-determination theory

Submetido: 29.03.2010 | Aceite: 08.03.2011

Rodrigo Batalha Silva, Thiago Sousa Matias, Maick da Silveira Viana e Alexandro Andrade. Laboratório de Psicologia do Esporte e do Exercício - LAPE; Centro de Ciências da Saúde e do Esporte - CEFID; Universidade do Estado de Santa Catarina - UDESC, Florianópolis, Brasil.

Endereço para correspondência: Rodrigo Batalha Silva, Centro de Ciências da Saúde e do Esporte - CEFID, Rua Pascoal Simone, 358 - Coqueiros, CEP: 88080-350 Florianópolis - SC, Brasil

E-mail: rbatalhasilva@gmail.com 
Dentre os fatores fundamentais para uma boa qualidade de vida e prevenção de doenças, está a prática de atividades físicas (Alves, Montenegro, Oliveira, \& Alves, 2005). Nesse sentido, há evidências que o sedentarismo tem sido um dos maiores problemas de saúde pública das sociedades modernas, atingindo cada vez mais a população de adolescentes (Seabra, Mendonça, Thomis, Anjos, \& Maia, 2008). Nessa fase do desenvolvimento a prática de atividades físico-esportivas é fundamental, pois favorece a adoção de um estilo de vida ativo e saudável, hábito este que tende a permanecer na vida adulta (Azevedo Júnior, Araújo, Silva, \& Hallal, 2007; Perkins, Jacobs, Barber, \& Eccles, 2004).

Entretanto, estudos confirmam a tendência de redução nos níveis de atividade física durante a adolescência, e que essa redução se deve tanto a fatores biológicos (Sallis, 2000) quanto a fatores psicossociais (Seabra et al., 2008). Tal problemática tem preocupado pesquisadores de diferentes áreas, que buscam melhor compreender a aderência dos jovens às práticas de exercícios físicos e desta forma intervir mais positivamente na busca de um estilo de vida mais ativo para esta população.

Embora a prática de exercícios físicos seja entendida pela população em geral como um dos principais fatores para manutenção da saúde (Siqueira et al., 2009), uma grande parcela não a pratica com a mínima frequência recomendada, o que demonstra a complexidade dessa questão. Nos domínios da Psicologia do Esporte e do Exercício a motivação tem sido um dos principais temas pesquisados (Gomes, Coimbra, Garcia, Miranda, \& Barra Filho, 2007), pois tem em sua essência o estudo das regulações que impedem ou facilitam a prática de comportamentos (Ryan \& Deci, 2000). As investigações conduzidas nessa área ao longo das últimas décadas têm como principal objetivo compreender os fatores sociais e intraindividuais que inibem ou facilitam a motivação para a prática de exercícios físicos (Blanchard, Mask, Vallerand, Sablonnière, \& Provencher, 2007).

$\mathrm{Na}$ atualidade, especialmente durante a última década, vem se destacando a Teoria da Autodeterminação (TAD; Deci \& Ryan, 1985) como uma possibilidade mais detalhada para o estudo dos aspectos motivacionais que envolvem a prática de exercícios físicos. Com a TAD, Deci e Ryan introduzem uma subteoria denominada Teoria da Integração do Organismo, estabelecendo a motivação de maneira contínua, caracterizada por diferentes níveis de autodeterminação (regulações motivacionais). Este continuum inclui a motivação intrínseca, a motivação extrínseca e suas diferentes regulações, e a amotivação, respectivamente da mais para a menos autodeterminada (Figura 1).

Esclarecendo a Figura 1, na extrema esquerda encontra-se a amotivação, que é um estado caracterizado pela falta de intenção, se associando assim à ausência de motivação. Nesse caso, a pessoa não percebe os motivos para o início ou continuidade da atividade

\begin{tabular}{|c|c|c|c|c|c|c|}
\hline \multirow{3}{*}{$\begin{array}{c}\begin{array}{c}\text { Tipo de } \\
\text { Motivação }\end{array} \\
\text { Tipos de } \\
\text { Regulação }\end{array}$} & \multicolumn{4}{|c|}{ Não Autodeterminado } & \multicolumn{2}{|c|}{ Autodeterminado } \\
\hline & Amotivacão & \multicolumn{4}{|c|}{ Motivação Extrínseca } & Motivação \\
\hline & $\begin{array}{c}\text { Sem } \\
\text { Regulação }\end{array}$ & $\begin{array}{l}\text { Regulação } \\
\text { Externa }\end{array}$ & $\begin{array}{l}\text { Regulação } \\
\text { Introjetada }\end{array}$ & $\begin{array}{l}\text { Regulação } \\
\text { Identificada }\end{array}$ & $\begin{array}{l}\text { Regulação } \\
\text { Integrada }\end{array}$ & $\begin{array}{c}\text { Regulação } \\
\text { Interna }\end{array}$ \\
\hline $\begin{array}{c}\text { Processo } \\
\text { Reguladores }\end{array}$ & $\begin{array}{c}\text { Não } \\
\text { intencional, } \\
\text { não } \\
\text { valorizado }\end{array}$ & $\begin{array}{c}\text { Recompensas, } \\
\text { castigos } \\
\text { externos }\end{array}$ & $\begin{array}{c}\text { Recompensas, } \\
\text { punições } \\
\text { internas }\end{array}$ & $\begin{array}{c}\text { Importância } \\
\text { pessoal, } \\
\text { valorização }\end{array}$ & Consciência & $\begin{array}{c}\text { Prazer, } \\
\text { satisfação }\end{array}$ \\
\hline
\end{tabular}

Figura 1. Continuum da Autodeterminação mostrando os tipos de motivações com seus tipos de regulação e os processos reguladores correspondentes (adaptado de Decy \& Ryan, 2000) 
(Fernandes \& Vasconcelos-Raposo, 2005). A seguir no quadro, à direita da amotivação, está a mais externa das motivações extrínsecas, a regulação externa. Esta é a regulação em que a pessoa é influenciada por fatores externos, executando a ação para receber recompensas ou evitar punições (Deci \& Ryan, 2000). Na sequência, mais à direita, encontra-se a regulação introjetada, onde a regulação envolve situações conflituosas e os comportamentos são influenciados por pressões internas, como a culpa e a ansiedade (Fernandes \& Vasconcelos-Raposo, 2005). O próximo estágio, mais interno, é a regulação identificada, onde o indivíduo considera a atividade importante e aprecia os resultados e benefícios que ela pode lhe proporcionar (Deci \& Ryan, 2000). A forma mais autodeterminada da motivação extrínseca é a regulação integrada, a qual não envolve só a identificação para a importância da atividade, mas também a integração dessas identificações com sua maneira de pensar, contendo assim algumas características da motivação intrínseca (Ryan \& Deci, 2000). Finalizando, na extrema direita do continuum está a motivação intrínseca, onde a atividade é praticada por conta do prazer, da satisfação e do divertimento que ela proporciona. Nesse caso, a regulação é exclusivamente interna, sem qualquer tipo de influência externa (Murcia \& Coll, 2006).

Teoricamente, pessoas mais autodeterminadas estão mais propensas a se envolverem em determinados comportamentos do que as com baixa autodeterminação (Deci \& Ryan, 2000). No campo da Psicologia do Esporte e do Exercício tais pressupostos têm se confirmado, pois pesquisas verificam que quem é mais autodeterminadas para a prática de exercícios físicos tem maior participação nestas atividades (Fernandes, Vasconcelos-Raposo, Lázaro, \& Dosil, 2004; Brickell \& Chatzisarantis, 2007; Edmunds, Ntoumanis, \& Duda, 2006; Ntoumanis, 2005). Sendo assim, conhecer a motivação dos adolescentes para a prática de exercícios físicos é fundamental na tentativa de fundamentar o estudo e a adaptação dos modelos de intervenção mencionados na literatura, levando em consideração as características do contexto social brasileiro.

Embora a TAD tenha sido publicada a mais de duas décadas e seja amplamente estudada no campo dos esportes e exercícios físicos em pesquisas realizadas na América do Norte, Europa e Oceania, só recentemente ela tem sido aplicada em pesquisas com populações brasileiras (Viana, Andrade, \& Matias, 2010, Viana, 2009; Vissoci, Vieira, Oliveira, \& Vieira, 2008), o que demonstra a necessidade de novos estudos e justifica a realização de pesquisas nessa área.

Considerando a relevância do conhecimento da motivação de adolescentes para a prática de exercícios físicos e a necessidade de estudos sobre a TAD aplicada a outras realidades ainda pouco conhecidas, este estudo objetivou investigar as relações entre a prática de exercícios físicos e suas regulações motivacionais, bem como os fatores associados a estas em estudantes adolescentes brasileiros.

\section{MÉTODO}

Trata-se de uma pesquisa descritiva de campo, do tipo transversal e correlacional (Thomas \& Nelson, 2002).

\section{Amostra}

Participaram do estudo 471 adolescentes com idades entre 14 e 18 anos, estudantes de uma escola pública estadual no município de Florianópolis/SC, Brasil. A amostra foi do tipo não-probabilística intencional. A instituição de ensino escolhida para a realização da pesquisa tem características especiais, pois abriga um grande número de alunos de diversas classes sociais e de diferentes cidades da região da Grande Florianópolis.

A maior parte da amostra é representada por estudantes do sexo feminino $(n=270$, $57.7 \%$ ), sendo que 3 adolescentes não informaram o sexo. A média de idade dos participantes foi de 15.9 anos, tanto para as meninas (15.9 $\pm 0.9)$ quanto para os meninos $(15.9 \pm 1.0)$. As classes socioeconômicas mais frequentes 
foram B1 e B2 para ambos os sexos. Quanto à classificação do IMC, meninas e meninos se diferenciaram, havendo maior prevalência de sobrepeso e obesidade entre os meninos (Tabela 1).

Quanto aos dados escolares, a maioria dos estudantes de ambos os sexos estavam matriculados no período vespertino. Entre as meninas predominaram as estudantes da segunda série, enquanto entre os meninos a primeira série do Ensino Médio foi a mais frequente (Tabela 1).

\section{Instrumentos}

Para a caracterização sociodemográfica da amostra foi utilizado um questionário baseado em Viana (2009). Foram investigadas informações referentes ao sexo, idade, peso, altura, série e turno de estudo. Foi ainda verificado o índice de massa corporal [IMC $=$ massa $(\mathrm{kg}) /$ altura $\left.^{2}(\mathrm{~m})\right]$ dos adolescentes. Este índice foi confrontado com os pontos de corte apresentados por Cole, Bellizzi, Flegal e Dietz (2000) e os adolescentes foram agrupados em grupos sem sobrepeso (eutróficos) e com sobrepeso (pré-obesidade e obesidade).

Para a classificação econômica foi utilizado o Critério Padrão de Classificação Econômica Brasil/2008 da Associação Brasileira de Empresas de Pesquisa - ABEP (2008). Esta tem como base os itens existentes na casa dos participantes (TV, geladeira, rádio, etc.) e o nível de escolaridade do chefe da família. A somatória dos pontos de cada questão resulta em um escore que representa as classes, agrupadas em três estratos arbitrados para este estudo: classe alta (A1, A2), classe média (B1 e B2) e classe baixa $(\mathrm{C} 1, \mathrm{C} 2, \mathrm{D})$. Os resultados de validação indicam que o questionário tem alta relação com a renda familiar $\left(r=.785\right.$ e $\left.r^{2}=62 \%\right)$.

Tabela 1

Frequência e percentual válido da classificação econômica (CE), classificação do IMC, série e turno de estudo de estudantes adolescentes da amostra geral, meninas e meninos

\begin{tabular}{lccc}
\hline & Geral & Meninas \\
$n(\%)$ & $n(\%)$ & Meninos \\
& $n(\%)$
\end{tabular}




\section{Prática de Exercícios Físicos}

O "Questionário de Avaliação da Atividade Física de Adolescentes" foi desenvolvido e validado por Florindo, Romero, Peres, Silva e Slater (2006) e escolhido por ser um instrumento desenvolvido para adolescentes brasileiros e por priorizar em sua avaliação a prática de exercícios físicos. O questionário tem 17 questões divididas em dois blocos: bloco 1) exercícios físicos (15 questões); e bloco 2) atividades físicas de locomoção à escola (2 questões). Ele avalia a atividade física anual do bloco 1 e semanal dos blocos 1 e 2, padronizando o questionário para gerar escores das atividades físicas em minutos/semana e minutos/ano. Para a presente pesquisa foram utilizados os escores anuais, por terem obtido melhores resultados em sua validação e contabilizarem apenas os exercícios físicos e esportes, desconsiderando as atividades de locomoção para a escola, pouco relevantes para esta pesquisa.

\section{Motivação para a Prática de Exercícios Físicos}

A motivação dos participantes para a prática de exercícios físicos foi avaliada utilizando o Questionário de Regulação de Comportamento no Exercício Físico-2 (Behavioral Regulation in Exercise Questionnaire-2 - BREQ-2; Markland \& Tobin, 2004). Tal questionário é baseado na TAD e tem o objetivo de quantificar as diferentes regulações motivacionais, interna e externas, bem como a amotivação, relacionadas à prática de exercícios físicos.

O questionário é composto por 19 itens do tipo Likert com 5 opções de resposta $(0=$ não é verdade para mim a $4=$ muitas vezes é verdade para mim), separadas em cinco diferentes construtos: amotivação (ex: "Acho que exercício é uma perda de tempo"), regulação externa (ex: "Faço exercícios porque outras pessoas dizem que devo fazer"), regulação introjetada (ex: "Sinto-me culpado/a quando não faço exercícios"), regulação identificada (ex: "Dou valor aos benefícios/vantagens dos exercícios") e motivação intrínseca (ex: "Gosto das minhas sessões de exercícios"). Utilizouse também o índice de autodeterminação, que é o escore obtido pela seguinte fórmula: $(-3 \times$ amotivação $)+(-2 \times$ regulação externa $)+$ $(-1 \times$ regulação introjetada $)+(2 \times$ regulação identifica $)+(3 \times$ regulação intrínseca $)$. O índice pode variar de -24 (menor autodeterminação) a 20 (maior autodeterminação).

Os testes de consistência interna da escala original obtiveram valores para o alpha de Cronbach superiores a .73, o que demonstra boa consistência interna para as diferentes subescalas do instrumento. Para este estudo foi utilizada a versão brasileira do BREQ-2 apresentada por Viana (2009), o qual obteve índices de confiabilidade variando entre .62 e .82 .

\section{Procedimentos}

Este estudo foi submetido e aprovado pelo Comitê de Ética em Pesquisas em Seres Humanos da Universidade do Estado de Santa Catarina (n. ${ }^{\circ}$ do protocolo 134/09). Considerando as normas éticas, os estudantes, ou seus responsáveis quando o participante tinha menos de 18 anos, tiveram de assinar os Termos de Consentimento Livre e Esclarecido para participação na pesquisa.

As coletas foram feitas no segundo semestre de 2009, em sala de aula, sempre com a presença de dois pesquisadores responsáveis pela pesquisa. Como sugerido pela direção da instituição de ensino, foram utilizados para a coleta dos dados os horários das aulas de Educação Física. Os questionários foram entregues aos alunos e uma explicação sobre o modo de respondê-los foi realizada. Durante o preenchimento dos instrumentos os pesquisadores estiveram presentes para o esclarecimento de possíveis dúvidas na interpretação das questões.

\section{Análise Estatística}

O tratamento dos dados foi realizado em duas etapas. Na primeira etapa foi realizada a estatística descritiva, com verificação da distribuição dos dados, por meio das curtoses, assimetrias e do teste de Kolmogorov-Smirnov (K-S), e cálculos de frequências, percentuais, médias e desvios padrão. 
Em seguida realizou-se a estatística inferencial, onde se optou por análises não-paramétricas considerando a distribuição dos dados. Para as comparações de amostras independentes foram utilizados os testes de MannWhitney para comparação de dois grupos, e Kruskal-Wallis para comparação de mais de dois grupos. Para correlação dos dados escalares foi utilizada correlação de Spearman ( $\rho)$. O nível de significância estabelecido foi de $\alpha=.05$, para todas as análises $(p<.05)$.

Considerando que meninas e meninos se diferenciaram quanto às principais variáveis em estudo, prática de exercícios físicos e regulações motivacionais, as análises foram realizadas dividindo o banco de dados por sexo

\section{RESULTADOS}

A Tabela 2 apresenta os dados gerais relacionados à média, desvio padrão, assimetria, curtose e resultado do teste de K-S das variáveis dependentes do estudo. Em relação às regulações motivacionais, verificam-se médias mais elevadas para as regulações internas, contrastando com os baixos níveis de amotivação e regulação externa. Os dados de assimetria e curtose, elevados para aproximadamente a metade das variáveis $(\neq 0)$ sugeriram a utilização de estatísticas não-paramétricas, confirmadas pelos resultados do K-S $(p<.05)$.
Verificaram-se diferenças entre meninas e meninos em relação à prática de exercícios físicos e às regulações motivacionais (Tabela 3). Meninos praticaram significativamente mais exercícios físicos do que as meninas, com aproximadamente 8.000 minutos anuais a mais em média. Em relação às variáveis motivacionais, meninas apresentaram maior regulação introjetada e menor motivação intrínseca e índice de autodeterminação do que os meninos, resultados que demonstram que estes são mais autodeterminados para a prática de exercícios físicos do que aquelas.

A quantidade de exercícios físicos esteve relacionada às diferentes regulações motivacionais e índice de autodeterminação (Tabela 4). Porém, é possível perceber algumas diferenças entre os sexos. Enquanto para as meninas a motivação intrínseca foi a variável mais fortemente relacionada à quantidade anual de exercícios físicos, para os meninos esta esteve mais relacionada à regulação identificada. Observa-se ainda, em relação ao sexo, que nas meninas a prática de exercícios correlacionouse negativamente com amotivação e positivamente com a regulação introjetada. Nos meninos a prática de exercícios físicos correlacionou-se negativamente com a regulação externa.

As regulações motivacionais se correlacionaram coerentemente, havendo poucas diferenças entre as meninas e os meninos.

Tabela 2

Média (M), desvio padrão (DP), assimetria, curtose e K-S da quantidade anual de exercícios físicos, regulações motivacionais e indice de autodeterminação de estudantes adolescentes

\begin{tabular}{lcccccc}
\hline & $n$ & $\mathrm{M}$ & $\mathrm{DP}$ & Assimetria & Curtose & K-S $(p)$ \\
\hline Minutos anuais de Exercícios & 415 & 14744 & 18448 & 1.86 & 3.63 & 0.000 \\
$\quad$ Físicos & 465 & 0.34 & 0.60 & 2.29 & 6.51 & 0.000 \\
Amotivação & 465 & 0.49 & 0.71 & 1.69 & 2.62 & 0.000 \\
Regulação Externa & 465 & 1.17 & 1.07 & 0.74 & -0.24 & 0.000 \\
Regulação Introjetada & 465 & 2.49 & 1.01 & -0.31 & -0.08 & 0.000 \\
Regulação Identificada & 465 & 2.73 & 1.12 & -0.64 & -0.43 & 0.000 \\
Motivação Intrínseca & 465 & 10.0 & 6.10 & -0.79 & -0.05 & 0.000 \\
Índice de Autodeterminação & & & & & &
\end{tabular}


Tabela 3

Média (M), desvio padrão (DP) da quantidade anual de exercícios físicos, regulações motivacionais e índice de autodeterminação de meninas e meninos estudantes adolescentes

\begin{tabular}{lcccccc}
\hline & \multicolumn{3}{c}{ Meninas } & \multicolumn{3}{c}{ Meninos } \\
& $n$ & M & DP & $n$ & M & DP \\
\hline $\begin{array}{l}\text { Minutos anuais de Exercícios } \\
\quad \text { Físicos ** }\end{array}$ & 233 & 11168 & 15641 & 179 & 19088 & 20084 \\
Amotivação & 265 & 0.37 & 0.66 & 197 & 0.31 & 0.50 \\
Regulação Externa & 265 & 0.50 & 0.71 & 197 & 0.45 & 0.70 \\
Regulação Introjetada * & 265 & 1.26 & 1.08 & 197 & 1.04 & 1.05 \\
Regulação Identificada & 265 & 2.41 & 1.07 & 197 & 2.58 & 0.93 \\
Motivação Intrínseca ** & 265 & 2.49 & 1.15 & 197 & 3.05 & 1.01 \\
Índice de Autodeterminação ** & 265 & 8.91 & 6.19 & 197 & 11.44 & 5.72 \\
\hline
\end{tabular}

Nota: ${ }^{*} p<.05,{ }^{* *} p<.001$

Tabela 4

Correlação ( $\rho$ ) entre a quantidade anual de exercícios físicos, regulações motivacionais e índice de autodeterminação de estudantes adolescentes (resultados das meninas na diagonal inferior e dos meninos na diagonal superior)

\begin{tabular}{lccccccc}
\hline & 1 & 2 & 3 & 4 & 5 & 6 & 7 \\
\hline $\begin{array}{l}\text { 1. Minutos anuais de Exercícios } \\
\quad \text { Físicos }\end{array}$ & - & -0.13 & $-0.15^{*}$ & 0.11 & $0.45^{* *}$ & $0.39^{* *}$ & $0.41^{* *}$ \\
2. Amotivação & $-0.16^{*}$ & - & $0.27^{* *}$ & 0.12 & $-0.29^{* *}$ & $-0.32^{* *}$ & $-0.57^{* *}$ \\
3. Regulação Externa & -0.11 & $0.32^{* *}$ & - & $0.23^{* *}$ & $-0.15^{*}$ & $-0.29^{* *}$ & $-0.51^{* *}$ \\
4. Regulação Introjetada & $0.24^{* *}$ & $-0.13^{*}$ & $0.16^{* *}$ & - & $0.37^{* *}$ & 0.05 & -0.11 \\
5. Regulação Identificada & $0.41^{* *}$ & $-0.23^{* *}$ & -0.01 & $0.46^{* *}$ & - & $0.54^{* *}$ & $0.68^{* *}$ \\
6. Motivação Intrínseca & $0.52^{* *}$ & $-0.31^{* *}$ & $-0.31^{* *}$ & $0.16^{* *}$ & $0.53^{* *}$ & - & $0.85^{* *}$ \\
7. Índice de Autodeterminação & $0.47^{* *}$ & $-0.57^{* *}$ & $-0.51^{* *}$ & 0.07 & $0.62^{* *}$ & $0.88^{* *}$ & - \\
\hline
\end{tabular}

Nota: ${ }^{*} p<.05,{ }^{* *} p<.01$

As regulações se relacionam positivamente com os construtos mais próximos, e negativamente com os mais distantes, demonstrando a existência de um continuum. Quanto à correlação das regulações motivacionais com o índice de autodeterminação, conforme o esperado as regulações externas se relacionam negativamente, e as mais internas positivamente com o índice (Tabela 4).

Quanto às variáveis associadas às regulações motivacionais das meninas (Tabela 5), verificou-se que as estudantes do grupo com maior idade e com presença de sobrepeso apresentaram maior regulação identificada. Em relação ao turno, as matriculadas no período matutino apresentaram maior regulação introjetada, enquanto as do período vespertino foram mais motivadas intrinsecamente $e$ apresentaram um maior índice de autodeterminação. Classe econômica e série de estudo não estiveram associadas às regulações motivacionais para as meninas.

Entre os meninos (Tabela 6), os estudantes com sobrepeso mostraram-se mais amotivados e menos motivados intrinsecamente do que os sem sobrepeso. Turno de estudo esteve associado ao índice de autodeterminação, sendo que os estudantes do período vespertino foram mais autodeterminados. Idade, classe econômica e série de estudo não estiveram associadas às regulações motivacionais para os meninos. 
Tabela 5

Regulações motivacionais e índice de autodeterminação $(M \pm D P)$ de meninas estudantes adolescentes em função da faixa etária, classes econômicas (CE), presença de sobrepeso, série e turno de estudo

\begin{tabular}{ccccccc}
\hline & $\mathrm{AM}$ & $\mathrm{REX}$ & $\mathrm{RIN}$ & $\mathrm{RID}$ & $\mathrm{MI}$ & $\mathrm{IA}$ \\
& $\mathrm{M} \pm \mathrm{DP}$ & $\mathrm{M} \pm \mathrm{DP}$ & $\mathrm{M} \pm \mathrm{DP}$ & $\mathrm{M} \pm \mathrm{DP}$ & $\mathrm{M} \pm \mathrm{DP}$ & $\mathrm{M} \pm \mathrm{DP}$ \\
\hline Faixa etária & & & & & & \\
14-15 & $0.43 \pm 0.60$ & $0.58 \pm 0.82$ & $1.13 \pm 1.04$ & $2.25 \pm 0.88^{* *}$ & $2.37 \pm 1.13$ & $8.02 \pm 6.16$ \\
$16-18$ & $0.35 \pm 0.69$ & $0.49 \pm 0.66$ & $1.33 \pm 1.09$ & $2.47 \pm 1.14^{* *}$ & $2.54 \pm 1.16$ & $9.19 \pm 6.25$ \\
CE & & & & & & \\
Alta & $0.63 \pm 0.94$ & $0.73 \pm 0.77$ & $1.27 \pm 0.97$ & $2.48 \pm 0.67$ & $2.67 \pm 1.00$ & $8.34 \pm 6.58$ \\
Média & $0.31 \pm 0.56$ & $0.47 \pm 0.63$ & $1.21 \pm 1.04$ & $2.38 \pm 1.07$ & $2.45 \pm 1.17$ & $9.05 \pm 6.10$ \\
Baixa & $0.40 \pm 0.82$ & $0.54 \pm 0.91$ & $1.40 \pm 1.25$ & $2.53 \pm 1.13$ & $2.72 \pm 1.02$ & $9.54 \pm 5.82$ \\
Sobrepeso & & & & & & \\
Não & $0.37 \pm 0.64$ & $0.51 \pm 0.73$ & $1.30 \pm 1.08$ & $2.46 \pm 1.01^{* *}$ & $2.56 \pm 1.10$ & $9.15 \pm 6.07$ \\
$\quad$ Sim & $0.44 \pm 0.63$ & $0.56 \pm 0.87$ & $1.31 \pm 1.24$ & $2.93 \pm 0.99^{* *}$ & $2.90 \pm 1.25$ & $10.79 \pm 6.24$ \\
Série & & & & & & \\
$1^{\circ}$ ano & $0.50 \pm 0.78$ & $0.57 \pm 0.81$ & $1.08 \pm 1.01$ & $2.20 \pm 1.08$ & $2.36 \pm 1.16$ & $7.77 \pm 6.68$ \\
$2^{\circ}$ ano & $0.30 \pm 0.57$ & $0.49 \pm 0.67$ & $1.42 \pm 1.17$ & $2.46 \pm 1.01$ & $2.59 \pm 1.09$ & $9.39 \pm 5.67$ \\
$3^{\circ}$ ano & $0.35 \pm 0.62$ & $0.46 \pm 0.68$ & $1.22 \pm 0.96$ & $2.60 \pm 1.12$ & $2.49 \pm 1.28$ & $9.47 \pm 6.52$ \\
Turno & & & & & &
\end{tabular}

Nota: ${ }^{*} p<.05,{ }^{* *} p<.01$

Tabela 6

Regulações motivacionais e indice de autodeterminação $(M \pm D P)$ de meninos estudantes adolescentes em função da faixa etária, classes econômicas (CE), presença de sobrepeso, série e turno de estudo

\begin{tabular}{|c|c|c|c|c|c|c|}
\hline & $\begin{array}{c}\mathrm{AM} \\
\mathrm{M} \pm \mathrm{DP}\end{array}$ & $\begin{array}{c}\mathrm{REX} \\
\mathrm{M} \pm \mathrm{DP}\end{array}$ & $\begin{array}{c}\text { RIN } \\
M \pm \mathrm{DP}\end{array}$ & $\begin{array}{c}\text { RID } \\
M \pm \mathrm{DP}\end{array}$ & $\begin{array}{c}\mathrm{MI} \\
\mathrm{M} \pm \mathrm{DP}\end{array}$ & $\begin{array}{c}\mathrm{IA} \\
\mathrm{M} \pm \mathrm{DP}\end{array}$ \\
\hline \multicolumn{7}{|l|}{ Faixa etária } \\
\hline $14-15$ & $0.37 \pm 0.59$ & $0.51 \pm 0.72$ & $1.07 \pm 0.97$ & $2.54 \pm 0.85$ & $2.99 \pm 1.02$ & $10.87 \pm 6.20$ \\
\hline $16-18$ & $0.27 \pm 0.44$ & $0.38 \pm 0.63$ & $0.99 \pm 1.09$ & $2.58 \pm 0.97$ & $3.09 \pm 1.00$ & $11.89 \pm 5.28$ \\
\hline \multicolumn{7}{|l|}{$\mathrm{CE}$} \\
\hline Alta & $0.33 \pm 0.57$ & $0.32 \pm 0.70$ & $1.04 \pm 1.20$ & $2.71 \pm 1.09$ & $2.84 \pm 1.30$ & $11.29 \pm 7.23$ \\
\hline Média & $0.29 \pm 0.48$ & $0.47 \pm 0.72$ & $0.99 \pm 1.02$ & $2.56 \pm 0.93$ & $3.09 \pm 0.98$ & $11.57 \pm 5.51$ \\
\hline Baixa & $0.41 \pm 0.66$ & $0.38 \pm 0.61$ & $1.37 \pm 1.11$ & $2.51 \pm 0.93$ & $2.84 \pm 1.07$ & $10.18 \pm 6.63$ \\
\hline \multicolumn{7}{|l|}{ Sobrepeso } \\
\hline Não & $0.27 \pm 0.45^{*}$ & $0.34 \pm 0.55$ & $1.07 \pm 1.11$ & $2.62 \pm 0.89$ & $3.16 \pm 0.92 *$ & $12.15 \pm 4.85$ \\
\hline Sim & $0.51 \pm 0.45^{*}$ & $0.68 \pm 0.97$ & $1.21 \pm 0.98$ & $2.60 \pm 1.04$ & $2.60 \pm 1.28^{*}$ & $8.92 \pm 7.89$ \\
\hline \multicolumn{7}{|l|}{ Série } \\
\hline $1^{\circ}$ ano & $0.36 \pm 0.56$ & $0.49 \pm 0.76$ & $1.06 \pm 1.05$ & $2.62 \pm 0.96$ & $3.10 \pm 1.05$ & $11.43 \pm 6.50$ \\
\hline $2^{\circ}$ ano & $0.33 \pm 0.52$ & $0.50 \pm 0.71$ & $0.91 \pm 0.91$ & $2.45 \pm 0.96$ & $2.90 \pm 1.01$ & $10.73 \pm 5.71$ \\
\hline $3^{\circ}$ ano & $0.14 \pm 0.30$ & $0.27 \pm 0.49$ & $1.05 \pm 1.20$ & $2.70 \pm 0.82$ & $3.28 \pm 0.80$ & $13.23 \pm 2.86$ \\
\hline \multicolumn{7}{|l|}{ Turno } \\
\hline Matutino & $0.41 \pm 0.65$ & $0.49 \pm 0.69$ & $1.00 \pm 0.95$ & $2.40 \pm 0.92$ & $2.86 \pm 1.07$ & $10.18 \pm 6.38^{*}$ \\
\hline Vespertino & $0.24 \pm 0.37$ & $0.40 \pm 0.66$ & $1.04 \pm 1.10$ & $2.67 \pm 0.93$ & $3.16 \pm 0.95$ & $12.25 \pm 5.03^{*}$ \\
\hline
\end{tabular}

Nota: ${ }^{*} p<.05,{ }^{* *} p<.01$ 


\section{DISCUSSÃO}

Este estudo teve como principal objetivo investigar as relações entre a prática de exercícios físicos e suas regulações motivacionais, bem como os fatores associados a estas em estudantes adolescentes de uma escola pública do município de Florianópolis/SC, Brasil. A motivação foi investigada com base na TAD (Deci \& Ryan, 1985), a qual para Ntoumanis (2001 como citado em Fernandes \& Vasconcelos-Raposo, 2005, p. 392) tem sido bem sucedida em sua aplicação no contexto da Educação Física.

Apesar da TAD ser bastante visada quando o assunto é motivação e prática de exercícios físicos, no Brasil ela pouco foi aplicada no campo da Educação Física e mais especificamente nas áreas da Psicologia do Esporte e do Exercício. A maior parte dos estudos publicados no país, sobre a temática, foram escritos por autores portugueses e espanhóis que investigaram populações européias (Fernandes \& Vasconcelos-Raposo, 2005; Murcia, Blanco, Galindo, Vullodre, \& Coll, 2007; Murcia \& Coll, 2006). Especificamente com adolescentes brasileiros, destacam-se três produções atuais. Duas que investigaram as relações da TAD e seus fundamentos com a prática de exercícios físicos na adolescência (Viana et al., 2010; Viana, 2009) e uma terceira que relacionou os pressupostos da TAD com a prática de exercícios físicos e a percepção de saúde mental dos adolescentes (Matias, 2010). Apesar das iniciativas com populações de adolescentes brasileiros, salienta-se a necessidade de diversificações das pesquisas, pois estas estão sendo conduzidas pelos mesmos pesquisadores e equipes de pesquisa.

Quanto à prática de exercícios físicos, os dados corroboram com outros estudos nacionais que apontam que a população de adolescentes do sexo masculino é mais ativa fisicamente do que a do sexo feminino (Silva, Nahas, Hoefelmann, Lopes, \& Oliveira, 2008; Souza \& Duarte, 2005; Vasques \& Lopes, 2009). Porém, é importante se considerar que pesquisas que diferenciam a prática de exercícios físicos em diferentes domínios (lazer, deslocamento, trabalho, doméstico, etc) podem apresentar resultados contrários a esta tendência em adolescentes. Del Duca et al. (2009), por exemplo, verificaram que mulheres eram mais sedentárias do que os homens nos ambientes de lazer e trabalho, mas praticavam mais atividades físicas nas atividades domésticas.

As diferenças entre os sexos não são exclusivamente biológicas, mas também influenciadas pelo contexto social. Há que se destacar ainda, como observado em Seabra et al. (2008), que a concepção de corpo, a qual está associada à prática de exercícios físicos muitas vezes não se enquadra no modelo de corpo feminino entendido como ideal pela sociedade. Neste caso, Seabra et al. (2008) evidencia as influências culturais, que privilegiam em muitos casos a prática de exercícios físicos para os meninos. Reflete-se então, até que ponto as interações culturais podem influenciar ou impedir na predisposição motivacional de meninas para a prática de exercícios físicos. No presente estudo, meninas apresentaram maior regulação introjetada, e menor motivação intrínseca e índice de autodeterminação do que os meninos. A literatura sugere que os meninos têm mais prazer ao praticar exercícios físicos, enquanto as meninas se pressionam mais internamente para a realização destas atividades (Markland \& Ingledew, 2007; Ntoumanis, 2005; Viana, 2009). Embora existam estudos que indicam o contrário, como Murcia, Gimeno e Coll (2007), os estudos revisados acima têm verificado que os meninos apresentam resultados superiores para as regulações motivacionais mais internas.

Viana (2009), ao comparar estudos realizados com população de adolescentes e adultos, observa que há uma tendência para que os meninos sejam mais autodeterminados que as meninas durante a adolescência, enquanto na vida adulta há evidências que as mulheres são mais autodeterminadas que os homens. Há que se considerar o fato de serem ainda limitadas as pesquisas brasileiras embasadas na TAD, e 
que a maior parte dos dados aqui discutidos são de pesquisas internacionais, sendo importante a realização de novos estudos sobre as diferenças na autodeterminação de adolescentes do sexo feminino e masculino. É fundamental compreender tais diferenças, tendo em vista que o fato de meninas e meninos terem um perfil motivacional diferente interfere sobre o modo com que os profissionais de Educação Física devem atuar junto a estes dois grupos.

As relações positivas da quantidade de exercícios físicos com as regulações mais internas sugerem que adolescentes mais autodeterminados praticam mais exercícios físicos do que os com baixa autodeterminação. Esses resultados corroboram com os pressupostos da TAD (Deci \& Ryan, 1985; Ryan \& Deci, 2000) e estudos recentes na área da Psicologia do Esporte e do Exercício com populações diversas (Brickell \& Chatzisarantis, 2007; Edmunds et al., 2006; Ntoumanis, 2005; Wilson \& Rodgers, 2004; Fernández et al., 2004). Vale destacar a pesquisa de Fernández et al. (2004), a qual investigou modelos teóricos da motivação para o contexto da Educação Física em adolescentes escolares. O estudo observou relações entre as diferentes regulações motivacionais e a intenção de praticar atividades físicas, porém a análise multivariada indicou que somente a motivação intrínseca predisse significativamente a intenção de se exercitar fora das aulas de Educação Física. Ainda segundo os autores, a relação entre exercício físico e motivação se mostra intimamente ligada a fatores sociais e a mediadores psicológicos. A partir de seus resultados, os mesmos sugerem que os profissionais de Educação Física devem centrar sua prática na promoção do aprendizado cooperativo, além de não deixar de possibilitar aos seus alunos um espaço para a escolha das atividades que mais lhe agrade.

Sob esses aspectos, é possível lançar mão dos conceitos relacionados às necessidades psicológicas básicas, construtos também presentes na TAD (Ryan \& Deci, 2000). Estudos empíricos têm verificado relações entre as necessidades psicológicas básicas, a motivação e os comportamentos frente aos exercícios físicos (Edmunds et al., 2006), de modo que quando supridas tais necessidades os indivíduos aumentam sua autodeterminação e consequentemente se exercitam mais. Com base nesse pressuposto, professores de Educação Física interessados em promover a prática de exercícios físicos entre seus alunos devem considerar: a importância da autonomia na prática, que não deve ser realizada como uma obrigação; a necessidade de os alunos se sintam competentes para desempenhar a atividade; e que sejam valorizados por outras pessoas ou a prática facilite a criação de vínculos sociais positivos. Uma abordagem que leve em consideração esses aspectos tornará o adolescente mais motivado, e consequentemente favorecerá a sua aderência aos exercícios físicos (Wilson, Rodgers, Blancharad, \& Gessel, 2003).

Meninas e meninos se diferenciaram em algumas das relações entre a prática de exercícios físicos e as regulações motivacionais. Para as meninas, a regulação introjetada se relacionou positivamente com a quantidade de exercícios físicos, o que não aconteceu para os meninos. Deste modo, as pressões internas são relevantes para as meninas e não para os meninos na aderência à prática de exercícios físicos. Por outro lado, a regulação externa esteve relacionada negativamente com a prática de exercícios físicos apenas entre os meninos, demonstrando que para eles as interferências externas podem ser prejudiciais à prática. Considerando o fato dos meninos serem mais autodeterminados, ou seja, têm mais prazer pela prática de exercícios físicos, espera-se que para eles as influências externas sejam mais prejudiciais, enquanto para as meninas o sentimento de obrigação também é importante na decisão de se envolver em uma prática, considerando que são menos autodeterminadas. Ressalta-se que estas relações nas quais meninas e meninos se diferenciaram foram fracas e, portanto, não conclusivas. 
As meninas com idade superior apresentaram maior regulação identificada do que as mais jovens, diferenças que não são observadas nas demais regulações motivacionais e entre os meninos. Em estudos também realizados com adolescentes, Murcia et al. (2007b) e Viana (2009) não verificaram relação entre idade e autodeterminação. Por outro lado, estudando indivíduos de diferentes faixas etárias, Murcia et al. (2007a) e Muyor, Águila, Silicia e Orta (2009) verificaram que os com maior idade eram mais autodeterminados para a prática de exercícios físicos do que os mais jovens. Esses resultados sugerem que grupos de idades próximas, como os adolescentes aqui investigados, tendem a não se diferenciar, ao contrário do que acontece quando comparados grupos de idades mais distantes. A realização de estudos que compreendam idades mais abrangentes podem melhor esclarecer essas diferenças, comparando jovens, adultos e idosos, por exemplo.

Adolescentes de diferentes classes econômicas, tanto meninas quanto meninos, apresentaram regulações motivacionais e índice de autodeterminação semelhantes. São incomuns os estudos que investigam as características socioeconômicas e suas relações com a autodeterminação para a prática de exercícios físicos. Parece importante ser considerado tal fator em estudos que envolvam o tema motivação, pois, como descrito na literatura, a motivação está diretamente associada às necessidades das pessoas (Deci \& Ryan, 2000; Feijó, 1998). Embora, assim como no estudo de Viana (2009), não se tenha observado diferenças na autodeterminação para a prática de exercícios físicos de estudantes de diferentes classes econômicas, devido à escassez de estudos fica em aberto essa área de investigação.

Apesar dos dados escolares também não receberem destaque na literatura da área, buscou-se verificar se adolescentes de diferentes séries e turnos de estudo apresentavam diferenças em suas regulações motivacionais. Adolescentes de diferentes séries apresentaram regulações motivacionais semelhantes. Quanto ao turno de estudo, os adolescentes matriculados no período vespertino estiveram mais autodeterminados. Considerando esses resultados, especificamente em relação ao turno e autodeterminação para a prática de exercícios físicos, estudos futuros que investiguem com maior profundidade esta questão podem obter resultados mais conclusivos, ainda não possíveis devido às poucas evidências científicas disponíveis na literatura sobre o tema.

Meninas com sobrepeso apresentaram maior regulação identificada do que as eutróficas, demonstrando estarem mais motivadas a praticar exercícios físicos por conta de seus benefícios. Entre os meninos, os eutróficos são mais motivados intrinsecamente e menos amotivados do que os com sobrepeso. No estudo de Viana (2009), não foram verificadas relações entre IMC e as diferentes regulações motivacionais. Por outro lado, em Markland e Ingledew (2007) a maior autodeterminação esteve associada a um baixo IMC tanto para meninas quanto para os meninos. Os resultados de Markland e Ingledew (2007) trazem ainda dados coerentes com os verificados no presente estudo, ao observar que meninos mais autodeterminados acreditavam ter proporções corporais abaixo de sua silhueta esperada, enquanto as meninas mais autodeterminadas acreditavam estar acima da sua silhueta ideal. Tais resultados sugerem uma relação complexa, onde mais do que as questões físicas ligadas à eutrofia ou ao sobrepeso, a imagem corporal é fundamental nessa relação com a autodeterminação para a prática de exercícios físicos.

\section{CONCLUSÕES}

Considerando os resultados e a discussão realizada, considera-se que a pesquisa obteve resultados que colaboram para o preenchimento de uma lacuna da Psicologia do Esporte e do Exercício no Brasil. Considerando o objetivo traçado, têm-se as seguintes conclusões: (i) Estudantes adolescentes mais autode- 
terminados praticam mais exercícios físicos, pois as regulações motivacionais mais externas se relacionam negativamente com a prática de exercícios físicos, enquanto as regulações mais internas se relacionam positivamente; (ii) As regulações motivacionais se relacionam positivamente com as mais próximas e negativamente com as mais distantes, confirmando o continuum da autodeterminação; (iii) Meninos são mais autodeterminados para a prática de exercícios físicos do que as meninas, além de praticarem mais tais atividades; e (iv) Faixa etária, sobrepeso e turno de estudo estão associados a algumas das regulações motivacionais, porém existem diferenças entre meninas e meninos.

Finalizando, é importante reforçar a existência de diferenças entre meninas e meninos nas relações entre as variáveis investigadas, o que não tem sido considerado em grande parte dos estudos da área da motivação. Essas diferenças devem ser levadas em consideração em estudos futuros que abordem o presente tema, pois a análise de meninas e meninos em conjunto pode mascarar importantes diferenças entre as duas populações.

\section{Agradecimentos:}

Os autores agradecem à comunidade da escola envolvida no estudo, à Secretaria de Educação do Estado de Santa Catarina e, por fim, aos contribuintes do estado que sustentam a UDESC.

\section{Conflito de Interesses:}

Nada a declarar.

\section{Financiamento:}

A pesquisa contou com o auxílio de bolsa de mestrado DS/CAPES.

\section{REFERÊNCIAS}

Alves, J. G., Montenegro, F. M., Oliveira, F. A., \& Alves, R. V. (2005). Prática de esportes durante a adolescência e atividade física de lazer na vida adulta. Revista Brasileira de Medicina do Esporte, 11 (5), 291-294. doi: 10.1590/S1517-86922005 000500009
Associação Brasileira de Empresas de Pesquisa ABEP (2008). Critério Padrão de Classificação Econômica Brasil/2008. Consultado em 18 de novembro de 2008, a partir de www.abep.org/ codigosguias/Criterio_Brasil_2008.pdf.

Azevedo Júnior, M. R., Araújo, C. L., Silva, M. C., \& Hallal, P. C. (2007). Continuidade na prática de atividade física da adolescência para a idade adulta: Estudo de base populacional. Revista de Saúde Pública, 41(1), 69-75. doi: 10.1590/S003 4-89102007000100010

Blanchard, C. M., Mask, L., Vallerand, R. J., Sablonnière, R., \& Provencher, P. (2007). Reciprocal relationships between contextual and situational motivation in a sport setting. Psychology of Sport and Exercise, 8(5), 854-873. doi: 10.101 6/j.psychsport.2007.03.004

Brickell, T. A., \& Chatzisarantis, N. L. D. (2007). Using self-determination theory to examine the motivational correlates and predictive utility of spontaneous exercise implementation intentions. Psychology of Sport and Exercise, 8(5), 758770. doi: 10.1016/j.psychsport.2006.11.001

Cole, T. J., Bellizzi, M. C., Flegal, K. M., \& Dietz. W. H. (2000). Establishing a standard definition for child overweight and obesity worldwide: International survey. British Medical Journal, 320, 1240-1243. doi: 10.1136/bmj.320.7244.1240

Deci, E., \& Ryan, R. (1985). Intrinsic motivation and self-determination in human behavior. New York: Plenum.

Deci, E., \& Ryan, R. (2000). The "what" and "why" of goal pursuits: Human needs and the selfdetermination of behavior. Psychological Inquiry, 11 (4), 227-268.

Del Duca, J. F., Rombaldi, A. J., Knuth, A. G., Azevedo, M. R., Nahas, M. V., \& Hallal, P. C. (2009). Associação entre nível econômico e inatividade física em diferentes domínios. Revista Brasileira de Atividade Física \& Saúde, 14(2), 123-131.

Edmunds, J., Ntoumanis, N., \& Duda, J. L. (2006). A test of self-determination theory in the exercise domain. Journal of Applied Social Psychology, 36(9), 2240-2265. doi: 10.1111/j.00 21-9029.2006.00102.x

Feijó, O. G. (1998). Corpo e movimento: Uma psicologia para o esporte. Rio de Janeiro: Shape.

Fernandes, H. M., \& Vasconcelos-Raposo, J. (2005). Continuum de auto-determinação: Validade para a sua aplicação no contexto desportivo. 
Estudos de Psicologia (Natal), 10(3), 385-395. doi: 10.1590/S1413-294X2005000300007

Fernandes, H. M., Vasconcelos-Raposo, J., Lázaro, J. P., \& Dosil, J. (2004). Validación y aplicación de modelos teóricos motivacionales en el contexto de la educación física. Cuadernos de Psicología del Deporte, 4(1/2), 67-89.

Florindo, A. A., Romero, A., Peres, S. V., Silva, M. V., \& Slater, B. (2006). Desenvolvimento e validação de um questionário de avaliação da atividade física para adolescentes. Revista de Saúde Pública, 40(5), 802-809. doi: 10.1590/S00 34-89102006005000002

Gomes, S. S., Coimbra, D. R., Garcia, F., Miranda, R., \& Barra Filho, M. (2007). Análise da produção científica em Psicologia do Esporte no Brasil e no exterior. Revista Iberoamericana de Psicologia del Ejercicio y el Deporte, 2(1), 25-40.

Markland, D., \& Ingledew, D. (2007). The relationships between body mass and body image and relative autonomy for exercise among adolescent males and females. Psychology of Sport and Exercise, 8(5), 836-853. doi: 10.1016/j. psychsport.2006.11.002

Markland, D., \& Tobin, V. (2004). A modification to the Behavioural Regulation in Exercise Questionnaire to include an assessment of amotivation. Journal of Sport and Exercise Psychology, 26, 191-196.

Matias, T. S. (2010). Motivação para a prática de atividade física relacionada aos estados de humor e de depressão na adolescência (Dissertação de mestrado não publicada). Universidade do Estado de Santa Catarina, Santa Catarina, Florianópolis, Brasil.

Murcia, J. A., \& Coll, D. (2006). A permanência de praticantes em programas aquáticos baseada na teoria da autodeterminação. Fitness $\mathcal{E}$ Performance Journal, 5(1), 5-9. doi: 10.3900/fpj.5.1.5.p

Murcia, J. A., Blanco, M. L., Galindo, C. M., Villodre, N. A., \& Coll, D. G. (2007). Efeitos do gênero, a idade e a frequência de prática na motivação e o desfrute do exercício físico. Fitness $\mathcal{E}$ Performance Journal, 6(3), 140-146. doi: 10.390 0/fpj.6.3.140.p

Murcia, J. A., Gimeno, E. C., \& Coll, D. (2007). Analizando la motivación en el deporte: Un estudio a través de la teoría de la autodeterminación. Apuntes de Psicología, 25(1), 35-51.

Muyor, J. M., Águila, C., Silicia, A., \& Orta, A. (2009). Análisis de la motivación autodeterminada en usuarios de centros deportivos.
Revista Internacional de Medicina y Ciencias de la Actividad Física y el Deporte, 9(33), 67-80.

Ntoumanis, N. (2005). A prospective study of participation in optional school physical education using a self-determination theory framework. Journal of Educational Psychology, 97(3), 444-453. doi: 10.1037/0022-0663.97.3.444

Perkins, D. F., Jacobs, J., Barber, B., \& Eccles, J. S. (2004). Fitness activities during young adulthood childhood and adolescent sports participation as predictors of participation in sports and physical. Youth Society, 35(4), 495-520. doi: 10.1177/0044118X03261619

Ryan, R., \& Deci, E. (2000). Self-determination theory and the facilitation of intrinsic motivation, social development, and well-being. American Psychologist, 55(1), 68-78. doi: 10. 103 7/110003-066X.55.1.68

Sallis, J. F. (2000). Age-related decline in physical activity: A synthesis of human and animal studies. Medicine $\mathcal{E}$ Science in Sports $\mathcal{E}$ Exercise, 32 (9), 1598-1600.

Seabra, A. F., Mendonça, D. M., Thomis, M. A., Anjos, L. A., \& Maia, J. A. (2008). Determinantes biológicos e sócio-culturais associados à prática de atividade física de adolescentes. Caderno de Saúde Pública, 24(4), 721-736. doi: 10.1590/S0102-311X2008000400002

Silva, K. S., Nahas, M. V., Hoefelmann, L. P., Lopes, A. S., \& Oliveira, E. S. (2008). Associações entre atividade física, índice de massa corporal e comportamentos sedentários em adolescentes. Revista Brasileira de Epidemiologia, 11(1), 159-168. doi: 10.1590/S1415-790X200800010 0015

Siqueira, F. V., Nahas, M. V., Facchini, L. A., Silveira, D. S., Piccini, R. X., Tomasi, E., ... \& Hallal, P. R. (2009). Fatores considerados pela população como mais importantes para manutenção da saúde. Revista de Saúde Pública, 43(6), 961-971. doi: 10.1590/S0034-89102009005000 066

Souza, G. S., \& Duarte, M. F. S. (2005). Estágios de mudança de comportamento relacionados à atividade física em adolescentes. Revista Brasileira de Medicina do Esporte, 11 (2), 104-108. doi: 10.1590/S1517-86922005000200002

Thomas, J. R., \& Nelson, J. K. (2002). Métodos de pesquisa em atividade física ( $3^{\mathrm{a}}$ ed.). Porto Alegre: Artmed.

Vasques, D., \& Lopes, A. (2009). Fatores associados à atividade física e aos comportamentos seden- 
tários em adolescentes. Revista Brasileira de Cineantropometria e Desempenho Humano, 11(1), 59-66.

Viana, M. S. (2009). Motivação de adolescentes para a prática de exercícios físicos: Perspectivas da teoria da autodeterminação (Dissertação de mestrado não publicada). Universidade do Estado de Santa Catarina, Santa Catarina, Florianópolis, Brasil.

Viana, M. S., Andrade, A., \& Matias, T. S. (2010). Teoria da autodeterminação: Aplicações no contexto da prática de exercícios físicos de adolescentes. Pensar a Prática, 13 (2), 1-18.

Vissoci, J. R., Vieira, L. F., Oliveira, L. P., \& Vieira, J. L. (2008). Motivação e atributos morais no esporte. Revista da Educação Física/UEM, 19(2), 173-182. doi: 10.4025/reveducfis.v19i2.5524

Wilson, P. M., \& Rodgers, W. M. (2004). The relationship between perceived autonomy support, exercise regulations and behavioral intentions in women. Psychology of Sport and Exercise, 5(3), 229-242. doi: 10.1016/S14690292(03)00003-7

Wilson, P. M., Rodgers, E. M., Blanchard. C. M., \& Gessel, J. (2003). The relationship between psychological needs, self-determined motivation, exercise attitudes and physical fitness. Journal of Applied Social Psychology, 33(11), 23732392.

(c) EY-NG Todo o conteúdo da revista Motricidade está licenciado sob a Creative Commons, exceto quando especificado em contrário e nos conteúdos retirados de outras fontes bibliográficas. 